\title{
Stabilität in der Währungsunion: Plädoyer für eine Staatsbank und eine Europäische Finanzagentur
}

\begin{abstract}
Die gegenwärtige Eurokrise bedroht den Fortbestand der Europäischen Währungsunion. Wodurch aber kommt es zu den Instabilitäten auf den Märkten für Staatsanleihen? Wie lassen sich solche Instabilitäten aus theoretischer Sicht erklären und wie könnten sie vermieden werden? Welche konkreten institutionellen Reformen sind auf europäischer Ebene notwendig, um die europäische Gemeinschaftswährung zu retten? Diesen Fragen widmet sich der folgende Beitrag.
\end{abstract}

\section{Einleitung}

Die Finanzkrise 2008 begann als eine Krise des privaten Finanzsektors. Infolge erheblicher Haushaltsdefizite, verursacht durch krisenbedingt verringerte Steuereinnahmen, zeitweilige Konjunkturprogramme und die Übernahme von Bankschulden, zusätzlich zu einer seit Jahren ungünstigen Entwicklung der Staatsschuldenstände in Relation zum Bruttoinlandsprodukt, verwandelte sie sich jedoch für viele Länder in eine Krise der Staatsfinanzen.

Die Krise stieß eine Diskussion an, zum Beispiel über die Verdienste der Fiskalpolitik und über die Grenzen staatlicher Haushaltsdefizite. In Europa drängte sich eine weitere Frage auf, die die Rolle der Fiskalpolitik in einer Währungsunion berührt. Die vorherrschende Meinung (Kenen 1969; De Grauwe 1994) lautet, dass Währungsunionen (insbesondere solche „nicht optimaler“ Natur) nicht ohne einen Finanzausgleichsmechanismus zwischen den Staaten auskommen. Der Grund dafür sei die Wahrscheinlichkeit asymmetrischer Schocks in den einzelnen Teilgebieten einer Union. Die Auswirkungen asymmetrischer Krisen ließen sich durch Fiskaltransfers dämpfen, die als Ersatz für Kapital- und Arbeitskräftemobilität wirken würden. In Europa fehlt ein solcher Mechanismus jedoch. Euroskeptiker argumentieren deshalb, Europa müsse einen Finanzausgleichsmechanismus schaffen, um die Gefahr eines zwangsweisen Austritts einiger Mitgliedsländer aus der Währungsunion zu bannen.

Der vorliegende Beitrag bietet eine andere Sichtweise der Eurokrise und verortet deren Ursache im Fehlen einer Staatsbank. Mit der Einführung einer gemeinsamen Währung und eines von der Europäischen Zentralbank (EZB) gesteuerten einheitlichen Wechselkurses löste Europa zwar das Problem der Währungsspekulation, ersetzte es aber gleichzeitig durch ein anderes: die Zinsspekulation auf dem Markt für Staatsanleihen. Denn mit der Einführung des Euro verzichteten die Mitgliedsländer auf ihre nationalen Notenbanken und deren Fähigkeit, Geld in Umlauf zu bringen, die Inflationsraten zu beeinflussen und die Staatsfinanzierung zu unterstützen.

Der vorliegende Beitrag stellt die These auf, dieser Konstruktionsfehler der gemeinsamen Währung könne durch die Einrichtung einer Europäischen Finanzagentur (EFA) behoben werden. Die EFA müsste befugt sein, nach eigenem Ermessen Anleihen aufzulegen, für die die Euromitgliedsländer gesamtschuldnerisch haften. Damit hätte die - für die Steuerung der monetären Rahmenbedingungen zuständige - EZB ein Pendant für die öffentliche Finanzwirtschaft.

\section{Neoliberale Wirtschafts- politik und ihr Einfluss auf den Euro}

Der Euro entstand in einer Zeit, in der neoliberale Theorien das politische Denken beherrschten. Neoliberale Konzepte treten für eine Beschneidung staatlicher Aufgaben und die Stärkung der Marktkräfte ein. In der Geldtheorie äußern sie sich in Aussagen wie:
- Fiskalpolitik bewirkt nichts;

- Geld ist neutral, eine Ausweitung der Geldmenge bewirkt nichts als Inflation;

- in der realen Wirtschaft kehrt als Reaktion auf negative Nachfrageschocks zügig und automatisch wieder Vollbeschäftigung ein.

Diese Hypothesen bestimmten den Aufbau der Europäischen Währungsunion und spiegeln sich sowohl in den rechtlichen Grundlagen der EZB als auch in deren Politik wider. Ausschlaggebend war vor allem die Entscheidung, eine direkte Einflussnahme der Zentralbank auf die Staatsfinanzierung strikt zu untersagen. Sie hatte eine Störung des Gleichgewichts zwischen Geld- und Fiskalpolitik zur Folge.

Solange die Länder ihre nationalen Währungen hatten, fiel ihren Zentralbanken auch hinsichtlich der öffentlichen Finanzwirtschaft eine Schlüsselrolle zu. Sie wirkten aktiv am Staatsschuldenmanagement mit, ermöglichten die Finanzierung von Haushaltsdefiziten und beteiligten sich an Rettungsmaßnahmen im Finanzsektor. Sie betätigten sich also als Staatsfinanzierungsbank. Die Architektur des Euroraums untergrub diese Rolle der nationalen Zentralbanken, indem sie

Thomas I. Palley, Dr., ist Leiter des
Projektes "Economics for Democratic and
Open Societies“ in Washington D.C. und
war zuvor Chefökonom der "US - China
Economic and Security Review Commission"
der US-Regierung. Arbeitsschwerpunkte:
Internationale Makroökonomie.
e-mail: mail@thomaspalley.com

Übersetzung aus dem Englischen von Lisa Eskuche 
ihnen das Recht nahm, Zahlungsmittel auszugeben. Dieser Umstand trug mit zu der Schuldenkrise bei, in der vor allem die sogenannten PIIGS-Länder (Portugal, Irland, Italien, Griechenland und Spanien) stecken. Die USA und das Vereinigte Königreich dagegen hatten ihre Federal Reserve beziehungsweise Bank of England und konnten daher die Zinssätze auf nahezu null drücken, Bailouts privater Finanzinstitutionen erleichtern und durch quantitative Lockerung, also den Aufkauf von Staatspapieren, ihre Budgetdefizite zu Niedrigstzinsen finanzieren. Damit gelang es diesen beiden Ländern, die Kosten der Krise für den öffentlichen Haushalt zu minimieren und zu vermeiden, dass aus der Schuldenkrise eine Vertrauenskrise wurde.

Nationale Währungssysteme verleihen den jeweiligen Regierungen die Kontrolle über den Anleihenmarkt. Die Architektur des Euro kehrt das Machtverhältnis um und legt die Zügel der nationalen Regierungen in die Hände des Marktes für Staatsanleihen. Angesichts der Vorherrschaft neoliberalen Denkens liegt der Schluss nahe, dass diese Folge der Konstruktion der gemeinsamen Währung beabsichtigt war.

Übersicht 1 zeigt die Unterschiede zwischen den geld- und fiskalpolitischen Strukturen verschiedener Länder. Zum Teil sind der Staatsverschuldung Grenzen gesetzt, zum Teil nicht. Die Zentralbanken können als Staatsbanken oder als vom Fiskalstaat abgekoppelte Institutionen agieren. Der entscheidende Unterschied besteht in diesem Fall darin, dass es der abgekoppelten Zentralbank nicht erlaubt ist, Staatspapiere aufzukaufen. Daraus ergibt sich auch die Abgrenzung gegenüber dem Begriff der unabhängigen Zentralbank: Unabhängig bedeutet in diesem Zusammenhang nur, dass die Zentralbank in ihren Entscheidungen nicht den Weisungen der Regierung unterliegt, sie aber dennoch befugt ist, Staatsschulden aufzukaufen.

Das moderne Papiergeldzeitalter (d. h. die Zeit seit der Aufgabe des Goldstandards in den 1930er Jahren) zeichnet sich durch eine Verbindung von „unbegrenzter Staatsverschuldung“ und „Staatsbank“ aus, die den derzeitigen Gegebenheiten in den USA und im Vereinigten Königreich entspricht. In den letzten Jahren haben manche Regierungen, darunter auch die britische, ihren Zentralbanken mehr Unabhängigkeit zugestanden; die Institutionen sind aber nicht abgekoppelt im oben dargestellten Sinne.
In den USA wird seit Langem gefordert, Budgetvorschriften zu erlassen, die einen ausgeglichenen Haushalt erzwingen. Mit einer solchen Regelung würden die USA in das rechte obere Feld der Übersicht 1 gehören. Das untere rechte Feld, in dem sich die Kriterien „Staatsverschuldung durch Vorschriften begrenzt" und „abgekoppelte Zentralbank” überschneiden, beschreibt faktisch die Situation vieler US-Staaten, die keine Zentralbank haben und der Forderung nach einem ausgeglichenen Haushalt nachkommen müssen. In dieses Feld gehören der derzeitigen Form nach auch die Euroländer. In diesen existieren zwar weiterhin die früheren Zentralbanken, allerdings nur dem Namen nach. Sie emittieren kein Geld mehr, sondern fungieren als Aufsichtsbehörden. Andererseits enthält der Vertrag von Maastricht eine Regelung zur Staatsverschuldung, die das Gesamthaushaltsdefizit der Länder auf $3 \%$ ihres Bruttoinlandsprodukts begrenzt. Die derzeitige Krise hat sich jedoch als so schwerwiegend erwiesen, dass man die Verschuldungskriterien des Maastrichter Vertrags weitgehend außer Acht gelassen und es geduldet hat, dass sich Länder des Euroraums über die Haushaltsvorschriften hinweggesetzt haben. Damit sind sie in das linke untere Feld der Grafik gerutscht.

\section{Abgekoppelte Zentralban-
ken und die Instabilität des Anleihenmarkts}

Auch wenn es (zumindest vorerst) hingenommen wird, dass Euroländer die Haushaltsvorschriften ignorieren, unterliegen sie dennoch der geldpolitischen Steuerung einer weisungsunabhängigen Zentralbank. Das Problem dabei liegt darin, dass es den Zentralbanken verwehrt ist, Staatsschulden aufzukaufen. Damit sind die Staatsanleihenmärkte dem Angriff der Spekulation schutzlos preisgegeben. Im Endeffekt ersetzte die Einführung des Euro also die

\begin{tabular}{lll|l}
\hline Übersicht 1: Vergleich geld- und fiskalpolitischer Strukturen \\
\hline & \multicolumn{2}{c}{ Haushaltsregeln } \\
& \multicolumn{2}{c}{ Staatsverschuldung unbegrenzt } & Staatsverschuldung begrenzt \\
\hline \multirow{3}{*}{ Zentralbank } & \multirow{2}{*}{ Staatsbank } & USA & USA: ausgeglichener Haushalt \\
& \multirow{2}{*}{ abgekoppelt } & UK & Eurgeschrieben \\
\cline { 2 - 3 } & ohne Maastricht-Regeln & US-Staaten \\
& & Euro-Länder mit Maastricht-Regeln \\
\hline
\end{tabular}

Devisenspekulation durch die Anleihenspekulation.

Das Problem der Rentenmarktspekulation lässt sich durch ein einfaches Modell darstellen: Investoren, die in Staatspapiere von Euroländern investieren, erwarten eine risikobereinigte Rendite in gleicher Höhe wie die Renditen, die sich mit risikofreien Anlagen erwirtschaften ließen. Dabei gehen sie von der Annahme aus, dass

(1) $1+i^{*}+z=E(R)$;

wobei gilt: $\mathrm{i}^{\star}=$ Zinssatz der risikolosen Anleihe, $z=$ Risikoprämie (Überrendite) für die Anlage in Staatsanleihen des betreffenden Landes, $\mathrm{E}(\mathrm{R})$ = erwartete Rendite der Anleihen des jeweiligen Landes.

Die Renditeerwartung für Anleihen einzelner Euroländer wird folgendermaßen berechnet:

(2) $E(R)=p(Y(d(i, .), i,. .),. i D / Y(d(i), i, \ldots), Z) X$

wobei gilt: $\mathrm{p}()=$. Ausfallwahrscheinlichkeit, $\mathrm{i}=$ Zinssatz des Landes, $\mathrm{d}=$ Haushaltsüberschuss/-defizit, D = Verschuldung im Verhältnis zum BIP, Z = Vertrauensvariable und $\mathrm{X}=$ Zahlung bei Nichtbedienbarkeit der Schulden. Für die Ausfallwahrscheinlichkeit gilt $0<\mathrm{p}<1$, für die Zahlung bei Ausfall $0<\mathrm{X}<1$.

Die Vorzeichen über den Funktionsargumenten bezeichnen die angenommenen Vorzeichen der partiellen Ableitungen. Ein Anstieg des Zinsniveaus im betreffenden Land bewirkt aufgrund des dadurch bedingten Anstiegs der Schuldendienstlast (iD) unmittelbar eine Erhöhung der Ausfallwahrscheinlichkeit. Die Auswirkungen auf Y heben sich dagegen zum Teil gegenseitig auf. Einerseits bewirkt ein höherer Zinssatz einen Rückgang der Privatnachfrage. Dem steht aber ein positiver Nachfrageeffekt der gestiegenen Zinszahlungen gegenüber, der sich über das Haushaltsdefizit äußert, denn Zinszahlungen sind Transferzahlungen. $+[1-p(Y(d(i), i, .),. i D / Y(d(i), i, \ldots), Z)][1+i] ;$ 
Abb. 1: Bestimmung der Zinssätze für Staatsanleihen

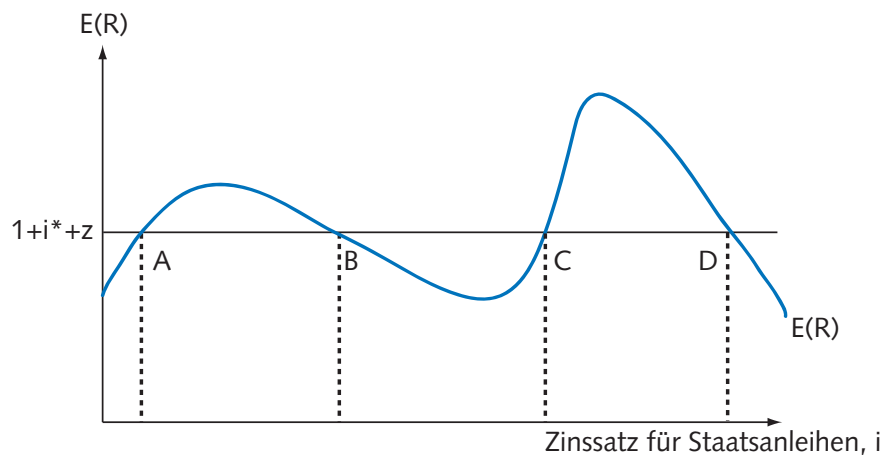

Quelle: Darstellung des Autors.

WSI MITTELIUNGEN

Abgeschlossen wird das Modell durch Hinzufügung eines dynamischen Zinsanpassungsmechanismus. Für diesen gilt:

(3) $d i / d t=f\left(1+i^{*}+z-E(R)\right) ; f(0)=0$, $f^{\prime}>0, f^{\prime \prime}>0$

Übersteigt die risikoangepasste Rendite risikofreier Anleihen die für Staatsanleihen eines Landes erwartete Rendite, so steigt der Zinssatz dieser Staatsanleihen, weil die Wertpapierhändler diese Staatsanleihen dann abstoßen und durch risikofreie Anleihen ersetzen.

Ausschlaggebend ist die Gleichung (2), welche die Entwicklung der Renditeerwartung beschreibt. Die erwartete Rendite kann aufgrund von Einbrüchen des Anlegervertrauens $(Z)$ schwanken und kann sich zudem im Verhältnis zum Zinssatz der Staatsanleihe nicht-linear entwickeln. Ökonomisch gesehen beruht die Logik der Nicht-Linearität auf miteinander konkurrierenden Effekten. Einerseits führen höhere Inlandszinssätze zu einem Anstieg der Renditeerwartung für Staatspapiere des entsprechenden Landes. Andererseits kann ein höheres Zinsniveau die Renditeerwartung aber auch drücken, da eine gestiegene Schuldendienstbelastung das Ausfallrisiko erhöht und höhere Zinsen sich negativ auf die Einkommen auswirken. Folglich kann sich in Anhängigkeit von der Sensitivität der p-Funktion und der Sensitivität von $Y$ gegenüber einem Anstieg von $i$ das Vorzeichen der Steigung der ER-Funktion umkehren.

Abbildung 1 stellt das Modell grafisch dar. Sie zeigt vier Gleichgewichtszustände: A ist das stabile „gute“ Gleichgewicht in Verbindung mit niedrigen Zinssätzen.
B bezeichnet ein unstabiles Gleichgewicht bei ebenfalls niedrigen Zinsen, $\mathrm{C}$ das „schlechte“ stabile Gleichgewicht bei hohem Zinsniveau und D ein unstabiles Gleichgewicht bei ebenfalls hohen Zinsen. In diesem Schema würden die PIIGS-Länder in den von hohen Zinssätzen geprägten negativen Gleichgewichtszuständen C oder D verharren. Die Herausforderung für die Politik besteht darin, sie von dort in den Bereich A zu befördern.

Im Kontext des Euroraums ist der risikofreie Anleihenzins, $i^{*}$ mit dem Zinssatz deutscher Bundesanleihen gleichzusetzen. Da Deutschland der größte und stärkste Wirtschaftsraum des Währungsgebiets ist, gelten die Anleihen des Bundes allgemein als die sichersten. Wie beim Geld (Menger 1892) könnte hier bis zu einem gewissen Grad eine sich selbst erfüllende Prophezeiung im Spiel sein: Da Bundesanleihen als vergleichsweise sicher gelten, ziehen sie die Anlagegelder an. Dadurch erhöht sich ihre Liquidität, gleichzeitig sinkt der Zinssatz, den der Bund zahlt. Die damit verbundene Verbesserung der Finanzlage Deutschlands untermauert weiterhin die Wahrnehmung der Bundesanleihen als risikofrei. Dadurch, dass Deutschland zum Sicherheitsstandard geworden ist, genießt das Land ein erstklassiges Ranking und die damit verbundenen fiskalischen Vorteile.

Der Umstand, dass in dem Modell mehrere Gleichgewichtszustände koexistieren, bedeutet, dass Hysterese auftreten kann. Nehmen wir an, dass Panikreaktionen auf dem Finanzmarkt für einen sprunghaften Anstieg der Risikoprämie (z) sorgen. Dann könnte es geschehen, dass die Staatsanleihen plötzlich von A (gutes Gleichgewicht bei niedrigen Zinsen) in den Bereich C oder D (schlechtes Gleichgewicht bei hohen Zin- sen) wechseln. Wenn die Panik vorüber ist und die Risikoprämie wieder fällt, verharrt das Land unter Umständen im Hochzinsgleichgewicht.

Eine ähnliche Situation kann eintreten, wenn das Vertrauen $(Z)$ in die künftige wirtschaftliche Performance eines Landes einbricht. In diesem Fall verschiebt sich die $E(R)$-Funktion nach unten. Ist die Verschiebung groß genug, kann der Inlandszins des betreffenden Landes in das schlechte Hochzinsgleichgewicht schnellen und anschließend dort verharren. Der Grund liegt darin, dass hohe Zinssätze eine self-fulfilling prophecy erhöhter Ausfallwahrscheinlichkeit erzeugen und die Anleger zum Ausgleich für das gestiegene Risiko höhere Zinssätze verlangen.

Der Fehler des Systems liegt darin, dass Regierungen, die spekulativen Angriffen ausgesetzt sind, sich nicht ihrer Zentralbank bedienen können, um in den Rentenmarkt einzugreifen und die Masse der umlaufenden Schuldtitel zu reduzieren. Ohne Zentralbank sind nationale Regierungen auf dem Rentenmarkt mit Provinzregierungen und Großunternehmen gleichgestellt, und damit sind sie der Spekulation und der Willkür des Marktes ebenso ausgesetzt wie jeder andere Kreditnehmer.

Die entsprechenden Bestimmungen in den Europäischen Verträgen untersagen der EZB jeden Eingriff zugunsten nationaler Staatsanleihen. Das Verbot ist auch durchaus angebracht, da Hilfen durch die EZB eine Vorzugsbehandlung einzelner Länder bedeuten und zu Fehlanreizen führen könnten. Könnten die Mitgliedsländer sich auf rettende Eingriffe der EZB verlassen, wäre die Versuchung groß, eine populistische Fiskalpolitik mit niedrigen Steuern und großen Haushaltsdefiziten einzuschlagen. Der politische Sog, dem die Gesamtheit der Mitgliedsländer dann ausgesetzt wäre, würde die Stabilität der Währung untergraben.

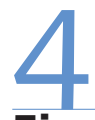

\section{Eine neue Architektur für Europas Staatsfinanzen}

Das Problem der Währungsunion liegt darin, dass in ihrem Aufbau keine Institution vorgesehen ist, die die nationalen Anleihenmärkte gegen Spekulationsangriffe verteidigen könnte. Dadurch sind die Regierungen in ihrer Fähigkeit ein- 
geschränkt, Haushaltsdefizite makroökonomisch optimal einzusetzen. Zudem sind die Bondmärkte damit willkürlicher Spekulation preisgegeben. Dieser Konstruktionsfehler ließe sich durch eine Neuordnung des staatlichen Finanzwesens und die Gründung einer Europäischen Finanzagentur (EFA) beheben, die Eurobonds mit gesamtschuldnerischer Haftung aller Mitgliedsländer ausgeben könnte. Der Dreh- und Angelpunkt dieser Lösung wäre allerdings das Recht der Europäischen Zentralbank, EFA-Anleihen zu handeln.

Die vorgeschlagene Architektur des öffentlichen Finanzwesens ruht auf drei Grundpfeilern (Abbildung 2). Erstens wären die Mitgliedsländer weiterhin berechtigt, eigene Staatsanleihen aufzulegen, für die sie ausschließlich selbst haften. Die Schuldtitel wären mit den Staatsanleihen der US-Bundesstaaten und mit Kommunalobligationen zu vergleichen und an eine Insolvenzordnung gebunden für den Fall, dass das betreffende Land in extreme finanzielle Schwierigkeiten gerät. Eine ähnliche Insolvenzordnung für Staatsanleihen wurde von Gianviti et al. (2010) vorgeschlagen. Das Insolvenzverfahren würde sich an die Regelungen in Chapter 9 des US-Konkursrechts anlehnen, die sich auf den Bankrott von Bundesstaaten und Kommunen beziehen. Sind sich die Märkte von vornherein dieser Möglichkeit bewusst, so unterliegt dieser Teil der Staatsfinanzierung der normalen Rentenmarktdisziplin.

Zweitens würden die Mitgliedsländer des Euroraums eine Europäische Behörde für Öffentliche Finanzen (EFA) gründen, die Anleihen mit gesamtschuldnerischer Haftung der Mitgliedsländer emittieren würde. Die EFA würde nach folgenden Regeln arbeiten:

(1) Die EFA wäre befugt, nach eigenem Ermessen Anleihen aller Laufzeiten aufzulegen.

(2) Der Erlös aus der Veräußerung dieser Anleihen würde den nationalen Regierungen zufließen.

(3) Die EZB wäre berechtigt, ausgegebene EFA-Bonds zu handeln.

(4) Die EFA stünde unter der Aufsicht der Finanzminister aller Euroländer als Vertreter der nationalen Regierungen.

\section{Abb. 2: Die vorgeschlagene Neuordnung des staatlichen Finanzwesens im Euroraum}

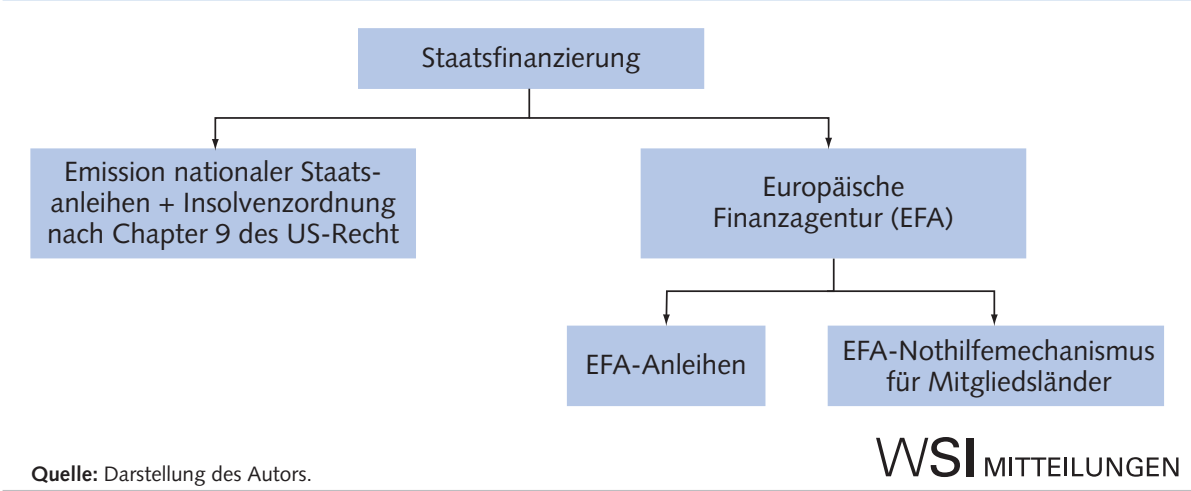

(5) Das Stimmrecht in der EFA würde in Abhängigkeit von den Bevölkerungszahlen ausgeübt.

(6) Die Erlöse aus dem Anleihenhandel würden ebenfalls nach der Einwohnerzahl verteilt, Gleiches gilt für den Zinsendienst der EFA-Anleihen.

Die EFA würde im Zusammenhang mit den von ihr emittierten Anleihen faktisch als Trust auftreten, der die Zinszahlungen der Länder einkassiert und sie an die Gläubiger weitergibt.

Der dritte Grundpfeiler des neuen Finanzgebäudes wäre die Aufsicht der EFA über einen Europäischen Krisenfond, der zahlungsunfähigen Mitgliedsländern mit Notdarlehen unter die Arme greifen könnte. Finanziert würde der Fond über den Verkauf von EFA-Anleihen. Wichtig ist in diesem Zusammenhang, dass die Gewährung von Mitteln aus dem Rettungsfonds an wirtschaftliche Auflagen gebunden wäre, die von der EFA verhängt würden. Der Krisenfonds wäre somit eine Art europäischer Internationaler Währungsfonds (IWF) (Palley 2010; Gros/Mayer 2010). In der gegenwärtigen Krise war es falsch, dass Griechenland den IWF um finanzielle Hilfe gebeten hat. Politisch gesehen leidet dadurch das Ansehen des Euro auf der Weltbühne, wirtschaftlich betrachtet war das Ersuchen unnötig. Der IWF kann keine Euro produzieren, Euro werden ausschließlich vom Währungssystem der Eurozone geschaffen. Folglich kann Europa seine eigenen Bailouts ohne die Hilfe des IWF durchführen. Die auf IWF-Darlehen $\mathrm{zu}$ entrichtenden Zinsen bedeuten den Abfluss von Steuergeldern aus dem europäischen Wirtschaftsraum und sind daher in Zeiten ohnehin schwacher Nachfrage kontraproduktiv.

Die vorgeschlagene EFA würde mehrere entscheidende Vorteile bieten. Der erste und wichtigste: Es wären die Voraussetzungen für einen Euro-Bond ohne die geringste Spur einer nationalen Identität geschaffen. Eine solche Anleihe könnte von der EZB gehandelt werden, ohne gegen die No-Bailout-Klausel zu verstoßen und würde der Zentralbank die Möglichkeit von Offenmarktgeschäften eröffnen. Als Ergebnis dessen könnte die EZB die Rolle der europäischen Staatsfinanzierungsbank spielen, die im derzeitigen institutionellen Gefüge fehlt.

Zweitens wäre die EFA in der Lage, über die jährliche Ausgabe von Anleihen in Abhängigkeit von der gesamtwirtschaftlichen Lage des Euroraums die jährliche Neuverschuldung der Länder mitzufinanzieren. Alle Euromitglieder erhielten Zahlungen im Verhältnis zu ihrer Bevölkerungszahl. Ginge die erhaltene Zahlung über den Bedarf eines Landes hinaus, könnte das Land die Mittel zur Tilgung seiner Schulden verwenden oder durch den Aufkauf fremder Staatsschulden einen Staatsfonds aufbauen.

Drittens wäre aufgrund der Abhängigkeit von der Bevölkerungszahl ein beschränkter fiskalischer Transfereffekt über das Bevölkerungswachstum gegeben. Bei Ländern mit langsam wachsender Bevölkerung dürften die Schuldenverbindlichkeiten im Laufe der Zeit sinken, während sie bei rapide wachsender Einwohnerzahl voraussichtlich zunehmen. Deutschland mit seinem langsamen Bevölkerungswachstum würde von der Entwicklung profitieren, gleichzeitig aber andere Länder an der eigenen Kreditwürdigkeit teilha- 
ben lassen und ihnen damit helfen, ihre Finanzierungskosten zu senken. Die anderen Länder hätten also ebenfalls einen individuellen Vorteil. Zudem käme die Verbesserung der öffentlichen Finanzlage der Wirtschaft des gesamten Eurogebiets zugute.

Darüber hinaus wäre die Schaffung einer EFA dem politischen Ziel eines föderalistischen und demokratischen Europas dienlich, das vielerorts als ein wesentlicher Beweggrund für die Einführung des Euro zitiert wird. Die demokratischen Voraussetzungen wären durch die Stimmrechtsverteilung in der EFA gewährleistet, die eine einheitliche Behandlung der Bürger aller Mitgliedsländer garantieren würde. Föderalistisch wäre die EFA zudem dank ihres einheitlichen, für alle gleichermaßen geltenden Rahmens. Wie Nuti (2011) anmerkt, könnte sich daraus zu einem späteren Zeitpunkt ein umfassender fiskalischer Föderalismus mit Ausgleichszahlungen zwischen den Ländern entwickeln. Eine solche Weiterentwicklung ist aber als separate Frage zu sehen und wird von der künftigen politischen Stimmung in den Euroländern abhängen.

\section{Politische Aufgabenvertei- lung im EZB/EFA-System}

Mit einer EZB/EFA-Lösung gliche die finanzpolitische Struktur des Euroraums dem System der USA und des Vereinigten Königreichs. Wie die Federal Reserve und die Bank of England wäre die EZB für die Geldpolitik einschließlich der Festlegung der Zinssätze zuständig. Auch die Wechselkurse blieben in ihrer Verantwortung.
Die Aufgabe der EFA bestünde in der Emission von Anleihen der Währungsunion. Da alle Erlöse aus der Anleihenplatzierung in die Kassen der Mitgliedsländer flössen, verbliebe die Ausgabengewalt uneingeschränkt bei den einzelnen Regierungen.

Das Kernstück des Systems wäre die Befugnis der EZB, zum Zweck der Steuerung der monetären Bedingungen nach eigenem Ermessen EFA-Anleihen zu kaufen und zu verkaufen - mit anderen Worten, Offenmarktgeschäfte zu betreiben, was ihr mit nationalen Staatsanleihen zu Recht verwehrt ist. Mehr noch, sie sollte EFA-Anleihen aller Laufzeiten auf dem Offenmarkt handeln und damit die zeitliche Zinsstruktur beeinflussen dürfen.

Abbildung 3 zeigt die wichtigsten Aufgaben der EZB im Rahmen des vorgeschlagenen neuen Systems. Ihre Hauptaufgaben wären erstens die Geldpolitik und zweitens die Zinspolitik für den Euroraum. Die EZB würde nicht nur über Lombardkredite den Tagesgeldzins steuern, sondern die gesamte Zinsterminstruktur der EFA-Bonds beeinflussen. In Krisensituationen könnte sie auch auf QuantitativeEasing-Maßnahmen zurückgreifen. Die EFA-Anleihen würden in der Praxis den risikofreien Zins für das gesamte gemeinsame Währungsgebiet vorgeben und somit als Bezugsgröße für alle anderen Instrumente einschließlich der Bonds der nationalen Zentralbanken dienen. Nach diesem Muster funktioniert das Geldsystem der USA. Die dritte Aufgabe der EZB bestünde in der Bereitstellung einer Krisenfazilität, um Institutionen des Privatsektors aus Liquiditätsengpässen herauszuhelfen. Dieser Hilfsmechanismus entspräche den Darlehensfazilitäten, die die Federal Reserve auf dem Höhepunkt der Finanzkrise 2008/2009 eingerichtet hat.

Abb. 3: Geldpolitische Aufgaben der Europäischen Zentralbank

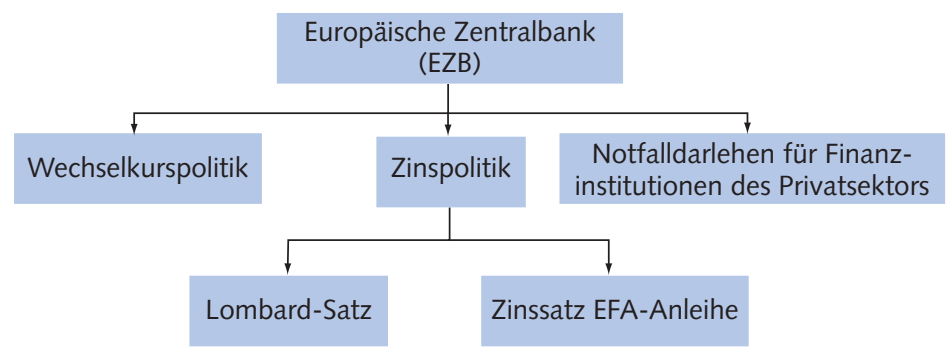

Quelle: Darstellung des Autors.

WSI MITTELUNGEN
Der Vergleich von Abbildung 2 und 3 zeigt eine symmetrische Aufgabenverteilung zwischen der vorgeschlagenen EFA und der EZB. Die EFA wäre im Wesentlichen für die Staatsfinanzierung zuständig. Sie würde Euro-Bonds ausgeben und eine Rettungsfazilität für Staaten bereitstellen. Die EZB würde dagegen die Zinssätze steuern und zu diesem Zweck als Europas Staatsbank fungieren. Zusätzlich würde sie dem privaten Finanzsektor bei Bedarf rettend unter die Arme greifen.

Da öffentliche Finanzpolitik und Geldpolitik Hand in Hand gehen sollten, wäre es sinnvoll, dass EZB und EFA im Rahmen dieser Aufgabenverteilung ihre jeweilige Politik abstimmen. Maßnahmen zur Finanzierung staatlicher Defizite durch die EFA sollten deshalb von der Einschätzung der monetären Erfordernisse durch die EZB abhängig gemacht werden und umgekehrt.

Ein entscheidender Vorteil der vorgeschlagenen Finanzarchitektur läge im Fehlen willkürlicher Einschränkungen wie der im Maastrichter Vertrag vorgeschriebenen maximalen Defizitquote von $3 \%$ des BIP. Diese und andere Beschränkungen haben sich politisch als ausgesprochen unpopulär erwiesen und unterhöhlen nach Meinung vieler die staatliche Souveränität und die demokratischen Strukturen. Zudem haben sie sich als nicht durchsetzbar erwiesen: Angesichts tief greifender wirtschaftlicher Schwierigkeiten haben manche Mitgliedsländer es vorgezogen, sich darüber hinwegzusetzen. Somit haben diese Vorschriften keinen Nutzen gebracht, sondern lediglich Kosten verursacht.

Natürlich bestünde ohne jeglichen einschränkenden Rahmen die Gefahr, dass einzelne Mitgliedsländer der Versuchung erliegen könnten, die Nachhaltigkeit ihrer Haushaltspolitik als zweitrangig zu betrachten. Die vorgeschlagene Finanzarchitektur sieht auf drei Ebenen Einschränkungen vor. Erstens kämen Finanzierungshilfen über die Auflage von EFA-Bonds nur infrage, wenn die Mehrheit der Bevölkerung der Eurozone durch ihre Finanzminister der Maßnahme zustimmt. Dies würde mehr Demokratie als bisher bedeuten, nicht weniger. Länder, die einer Ausgabe von Euro-Bonds nicht zugestimmt haben, könnten die ihnen zufallenden Erlöse auf die Tilgung ihrer eigenen Staatsschulden verwenden. Da die Staatsschuld mit einem höheren Risikorating behaftet ist als die Euro-Anleihen, würden die Länder mit 
der Senkung ihrer Schuldendienstverbindlichkeiten insgesamt einen Gewinn erwirtschaften.

Zweitens wäre der Rückgriff auf den staatlichen Rettungsfond nicht uneingeschränkt möglich. Um Mittel aus diesem Topf zu beziehen, müssten Hilfe suchende Länder politische Auflagen der EFA erfüllen. Auch diese Vorgaben würden auf demokratischem Wege festgelegt, da ihnen eine Mehrheit der Finanzminister im Rat der EFA zustimmen müsste. Auf der dritten Ebene, dem Handel mit nationalen Staatsanleihen, würde die Marktdisziplin eine regulierende Wirkung ausüben.

Durch diese Einschränkungen ließe sich die Haushaltsdisziplin ohne die gegenwärtigen negativen Auswirkungen auf die Staatsfinanzierung aufrechterhalten. Das jetzige System zieht durch die in den Europäischen Verträgen verankerte Regel, die es der EZB verbietet, Staatsschulden aufzukaufen, einen scharfen Trennstrich zwischen den Staatsfinanzen und der Zentralbank. Dadurch werden die nationalen Regierungen auf dem Rentenmarkt mit Provinzregierungen und Großunternehmen gleichgesetzt, mit katastrophalen Folgen.

Sobald sie voll funktionsfähig wäre, würde die EFA die Mitgliedsländer bei der laufenden Haushaltsfinanzierung unterstützen. Dem müsste jedoch eine Übergangsperiode vorangehen, in der die Länder ihre eigenen Anleihen gegen EFA-Bonds tauschen könnten. Der Übergang würde wie folgt ablaufen. Die EFA würde Anleihen platzieren und mit dem Erlös nationale Staatsanleihen im Verhältnis zur jeweiligen Bevölkerungsgröße aufkaufen. Die Staatsanleihen wären damit eingezogen, stattdessen würden die EFA-Mitgliedsländer im Verhältnis zu ihrer Bevölkerungszahl den Schuldendienst der EFA-Anleihen übernehmen. Länder mit geringer Staatsverschuldung wie Luxemburg bekämen zum Ausgleich der über ihren bisherigen Schuldendienst hinausgehenden Zinslast EFA-Anleihen gutgeschrieben.

\section{Vergleich mit dem "Blue Bond"}

Vor nahezu 20 Jahren machte Stuart Holland (1993) als wirtschaftlicher Berater von Jacques Delors den Vorschlag eines von einem Europäischen Investitionsfonds zu emittierenden Gemeinschaftsbonds als Vehikel zur Übertragung nationaler Staatsschulden auf die Europäische Union. Die Idee wurde zwar nicht aufgegriffen, feierte aber jüngst im Zusammenhang mit der Schuldenkrise in der Währungsunion eine Wiederauferstehung. ${ }^{1}$ Auch De Grauwe/Moesen (2009) schlagen eine Gemeinschaftsanleihe vor. Auf diesen Gedanken bauen Delpla/von Weizsäcker (2010) mit ihrem kürzlich vorgestellten Modell eines „Blue Bond” auf, den die Euro-Länder individuell ausgeben und für den andere Euro-Länder gesamtschuldnerisch haften würden. Dahinter steckt der Gedanke, hochverschuldete Mitgliedsländer an dem günstigeren Rating finanziell besser gestellter Länder teilhaben zu lassen, um ihre Zinsbelastung zu reduzieren und ihre Haushaltssituation verbessern zu können.

Ungeachtet scheinbarer Parallelen unterscheidet sich das hier beschriebene EFA-Modell in grundlegenden Aspekten vom Blue Bond. Die wichtigste Differenz besteht darin, dass im ersten Fall die EZB Anleihen der EFA handeln und damit die zeitliche Zinsstruktur des gemeinschaftlichen Währungsgebiets beeinflussen könnte. Warum macht das EFA-Modell dies möglich, aber nicht der Blue Bond? Das Emissionsverfahren der EFA-Bonds stellt sicher, dass alle Euroländer im Verhältnis zu ihrer Bevölkerungszahl einheitliche Finanzmittel erhalten, womit jegliche nationalstaatliche Anmutung vermieden wird. Aufkäufe von EFA-Bonds durch die EZB kämen allen Euroländern in mehr oder weniger gleichem Umfang zugute, und die EZB würde damit nicht gegen die No-Bailout-Klausel verstoßen. Im Gegensatz dazu würde im Fall des Blue Bond das einzelne Land darüber entscheiden, welche Anzahl solcher Anleihen es ausgeben will. Der jeweilige Blue Bond hätte zudem eindeutig nationalen Charakter. Von BlueBond-Aufkäufen durch die EZB würde folglich nur das Land profitieren, dessen Anleihen aufgekauft werden. Das BlueBond-Modell bricht also nicht mit dem derzeitigen System staatlicher Anleihen. Der einzige Unterschied besteht darin, dass diese Titel bis zu einem gewissen Prozentsatz von der Gemeinschaft gesichert wären.

Ein zweiter Vorteil des hier unterbreiteten Modells wäre, dass die EFA zur Unterstützung der normalen Haushaltsfinanzierung der Euroländer laufend Anleihen auflegen würde. Diese Anleihen sollen zu einem gängigen Instrument der staatlichen
Finanzierung werden. Das Blue-Bond-Modell dagegen wurde im Angesicht der Krise als reine Notmaßnahme erdacht. Es soll Ländern in finanzieller Bedrängnis unter die Arme greifen, indem es sie in gewissem Umfang am deutschen Rating teilhaben lässt. Damit macht es ihnen den Umtausch hochverzinslicher Anleihen gegen Schuldtitel mit niedrigerem Zinssatz möglich.

Der dritte Vorteil des EFA-Vorschlags wäre, dass er völlig auf Restriktionen nach dem Muster des Maastrichter Vertrags mit seiner 3-\%-Klausel verzichtet. Die Haushaltsdisziplin wäre durch den demokratischen Entscheidungsprozess innerhalb der EFA sowie über die Marktdisziplin gewährleistet. Im Gegensatz dazu behält das Modell des Blue Bond die Maastrichter Defizitrestriktionen bei und erlegt den Ländern eine Wertgrenze auf, die die Möglichkeiten der Emission von Blue Bonds einschränkt (im Gespräch sind $60 \%$ des Bruttoinlandsprodukts).

Um es zusammenzufassen, geht das EFA-Modell über den Blue Bond hinaus. Es zielt darauf ab, das fiskalische Gefüge des Euroraums umzugestalten, indem es der Europäischen Zentralbank wieder die Rolle einer Staatsbank zuerkennt. Durch das Abstreifen des neoliberalen Designs, das die EZB strikt von der staatlichen Finanzpolitik trennt, käme faktisch eine grundlegende Neuordnung der Währungsunion zustande. Wie das Modell des Blue Bond verschafft auch die vorgeschlagene EFA krisenbefallenen Ländern durch die Bereitstellung von Anleihen zu günstigeren Bedingungen Zinserleichterungen. Gleichzeitig beseitigt es aber alle undemokratischen und gegen die staatliche Souveränität verstoßenden Restriktionen á la Maastricht. Dazu eröffnet es der EZB die Möglichkeit, als Staatsfinanzierungsbank tätig zu werden und EFA-Anleihen am Offenmarkt zu handeln.

\section{Schlussfolgerungen}

Ich habe den Sachverhalt aus theoretischer und politischer Sicht erörtert. Auf der the-

\footnotetext{
1 Ich bin Mario Nuti zu Dank verpflichtet, weil e mich auf den Zusammenhang zwischen dem Vorschlag von Holland und den aktuellen Erwägungen aufmerksam machte.
} 
oretischen Ebene geht es um die Stabilität von Währungsunionen und die Notwendigkeit einer Staatsbank zur aktiven Beeinflussung des Anleihenmarkts. In seinem politischen Ansatz habe ich einen spezifischen Vorschlag zur Stabilisierung des Euro durch die Schaffung einer Staatsbank für den gemeinsamen Währungsraum beschrieben.

Es zeichnet sich immer deutlicher $a b$, dass das derzeitige institutionelle Gefüge der gemeinsamen Währung nicht stabil genug ist. Es ist abzusehen, dass der Euro auseinanderbricht, wenn sich daran nichts ändert. Die Ursache der mangelnden Stabilität liegt in der hohen Zinsbelastung hochdefizitärer Länder, die zu einer auf Dauer nicht tragbaren Verschuldung führt. Das Problem lässt sich nur über eine Zentral- bank lösen, die als Staatsfinanzierungsbank eingreift und die Zinsen auf ein akzeptables Niveau drückt. Dies ließe sich mit einer Europäischen Finanzagentur erreichen.

Analytisch gesehen beschreibt der vorliegende Beitrag einen dritten Weg für den Euro. Der derzeitig eingeschlagene Weg, der Änderungen nach dem Vorbild des Blue Bond vorsieht, hält weiterhin an der Architektur von Maastricht mit ihren quantitativen Beschränkungen von Defizit und Verschuldung fest und ändert nichts an der Trennung von EZB und staatlicher Finanzwirtschaft. Ein zweiter Weg bestünde in einer fiskalischen Transferunion. Der hier aufgezeigte dritte Weg läuft auf eine umfassende Währungs- und Fiskalunion ohne quantitative Beschränkungen hinaus, sieht aber keinen Finanzausgleich vor.
In der Diskussion über die finanzwirtschaftliche Struktur der Währungsunion geht es nicht nur um wirtschaftliche Fragen. Die politischen Auswirkungen sollten nicht unterschätzt werden. Die derzeitige, durch neoliberales Denken geprägte EuroArchitektur mit ihrer strikten Trennung von Zentralbank und staatlicher Finanzwirtschaft setzt die Regierungen der Euroländer fortwährend einem zusätzlichen finanziellen Druck aus. Dieser Druck dürfte es mit der Zeit schwierig machen, die ausgebauten Wohlfahrtsstaaten Europas aufrecht zu erhalten. Es macht deshalb nicht nur wirtschaftlich, sondern auch politisch Sinn, die Währungsunion einer Reform zu unterziehen und eine Europäische Finanzagentur einzuführen.

\section{LITERATUR}

De Grauwe, P. (1994): The Economics of Monetary Integration, Oxford De Grauwe, P./Moesen, W. (2009): Gains for all: a Proposal for a Common Eurobond, in: Intereconomics 44 (3), S. 132-135

Delpla, J./von Weizsäcker, J. (2010): The Blue Bond Proposal, Bruegel Policy Brief (3), hrsg. vom Bruegel Institut, Brüssel

Gianviti, F./Krueger, A.O./Pisani-Ferry, J./Sapir, A./Hagen, J. von (2010): A European Mechanism for Sovereign Debt Crisis Resolution: a Proposal, hrsg. vom Bruegel Institut, Brüssel

Gros, D./Mayer, T. (2010): Disciplinary Measures, in: The Economist vom 18. Februar

Holland, S. (1993): The European Imperative: Economic and Social Cohesion in the 1990s, Foreword by Jacques Delors, Nottingham
Kenen, P. (1969): The Theory of Optimum Currency Areas: An Eclectic View, in: Mundell, R./Swobodaa, A. (Hrsg.): Monetary Problems of the International Economy, Chicago

Menger, K. (1892): On the Origin of Money, in: Economic Journal 2

(6), S. 239-255

Nuti, D. M. (2011): Euro Sovereign Debt: Would a Single European Bond Work?, http://dmarionuti.blogspot.com/2011/02/singleeuropean-sovereign-bond-pie-in.html Palley, T. I. (2010): Euroland is Being Crucified Upon Its Own Cross of Gold, FT Economists' Forum vom 10. Februar 